\title{
Linearização por realimentação granular robusta com algoritmo evolutivo Takagi-Sugeno: Análise e avaliação de desempenho *
}

\author{
Anderson Bento* Lucas Oliveira* Valter Leite* \\ Fernando Gomide** \\ * Departamento de Engenharia Mecatrônica, CEFET-MG, Divinópolis, \\ MG, Brasil (e-mail: bentoavb@gmail.com, lqsoliveira@cefetmg.br e \\ valter@ieee.org). \\ ** Departamento de Engenharia da Computação e Automação, \\ FEEC-UNICAMP, Campinas, SP, Brasil (e-mail: \\ gomide@dca.fee.unicamp.br).
}

\begin{abstract}
Feedback linearization is a powerful nonlinear control technique, but its performance degenerates substantially when subject to modeling errors. Recently robust granular feedback linearization, a method that uses the participatory learning to cancel the effect of model mismatches, was introduced to reduce the impact of modeling errors in the control loop. This paper aims to evaluate the performance of the robust, granular feedback linearization controller with learning done using eTS, the evolving Takagi-Sugeno approach. The behavior of the robust, granular feedback linearization with eTS is evaluated using an inverted pendulum control system to track a specified trajectory. Performance is measured using integral of the absolute error, the integral of the variability of the control signal, and the integral of the variability of the error indexes. Simulation results suggest that the robust, granular eTS control performs better than exact feedback linearization.

Resumo: A linearização por realimentação é uma poderosa técnica de controle não linear, mas quando aplicada a situações em que há erro de modelagem, seu desempenho fica comprometido e o comportamento em malha fechada degenera. A linearização por realimentação granular robusta é uma técnica que utiliza aprendizagem participativa para cancelar os efeitos causados por erros de modelagem na malha de controle. Esse trabalho objetiva avaliar o desempenho do controlador de linearização por realimentação granular robusta quando o mecanismo de aprendizagem é substituído pelo eTS, o algoritmo evolutivo Takagi-Sugeno. O controlador granular robusto com eTS é utilizado para que os estados de um pêndulo invertido siga uma trajetória especificada. Os resultados de simulação são quantificados usando os critérios da integral do valor absoluto do erro, da integral da variabilidade do erro e da integral da variabilidade do sinal de controle. Os resultados sugerem que o controlador granular robusto com eTS tem desempenho superior à linearização por realimentação exata.
\end{abstract}

Keywords: Feedback linearization; evolving systems; adaptive control; granular computing. Palavras-chaves: Linearização por realimentação; sistemas evolutivos; controle adaptativo; computação granular.

\section{INTRODUÇÃO}

A linearização por realimentação exata (EFL - exact feedback linearization) é um potente e importante método de controle aplicável a uma classe de processos não lineares (Khalil, 2002). Esse método propõe cancelar total ou parcial as não linearidades presentes na dinâmica do processo (Ataei et al., 2005), o que exige um conhecimento preciso das funções que descrevem o comportamento do processo e assim possibilitar o cancelamento exato das não linearidades (Sastry, 1999). Contudo, em muitas situações

\footnotetext{
^ Os autores agradecem ao CEFET-MG, FAPEMIG e CNPq pelo apoio financeiro.
}

é inviável obter um modelo preciso do processo, seja pela impossibilidade de se obter os valores exatos dos parâmetros, ou pelo fato de não se considerar todos os fatores para descrever o comportamento do processo exatamente. Esses fatos levam a um modelo impreciso e uma lei de controle não linear que é incapaz de tratar a diferença entre a verdadeira dinâmica do processo e a do modelo, o que causa problemas que podem instabilizar o sistema em malha fechada (Guillard and Boulès, 2000).

$\mathrm{Na}$ literatura encontram-se diversas abordagens e esquemas que buscam agregar robustez ao EFL. Por exemplo, (Sastry and Isidori, 1989) apresentam uma técnica adaptativa de controle para sistemas não lineares com fase mí- 
nima. Baseando-se na teoria de sistemas fuzzy, (Wang and Mendel, 1992) desenvolveram um algoritmo usando princípio dos mínimos quadrados recursivos para construir uma base de regras fuzzy. Conforme (Park and Park, 2003), as propriedades desse algoritmo possibilitaram que outros autores desenvolvessem arquiteturas de controle adaptativas fuzzy para sistemas não lineares (Souza et al., 2012), (Silva et al., 2013), (Leite et al., 2015). Nessa linha têm-se, por exemplo, o controle adaptativo fuzzy indireto que estima a dinâmica e o sinal de controle da malha fechada (Wang, 1996), (Banerjee et al., 2011), (Park et al., 2003). Por outro lado, existe um grupo especial de modelos baseados em regras fuzzy denoninado modelos fuzzy do tipo TakagiSugeno - TS (Sugeno and Takagi, 1983). Modelos TS são modelos fuzzy funcionais que tem sido usados para agregar robustez ao EFL conforme sugere (Yang et al., 2004). Contudo, em meados da década de 2000, (Angelov and Filev, 2004) modificaram o algoritmo TS para atualizar uma base de regras existente e/ou criar novas regras em tempo real, a partir do uso de métodos de agrupamento de dados recursivos. Essa técnica, denominada algoritmo evolutivo Takagi-Sugeno (evolving Takagi-Sugeno - eTS), tem sido usada em diversos trabalhos de identificação de sistemas não lineares (Precup et al., 2017). Recentemente (Lima et al., 2010) propôs o algoritmo de aprendizagem participativa (evolving participatory learning - ePL). Assim como no algoritmo eTS, o ePL baseia-se no conceito de agrupamento recursivo para atualizar e criar regras. Porém, o ePL difere do eTS no processo de atualização da base de regras, uma vez que no ePL o valor modal da função de pertinência no antecedente de uma regra não corresponde necessariamente a um dado de entrada. Além disso, o ePL verifica e remove regras redundantes, além de ser menos sensível a outliers. O ePL foi utilizado em um sistema de controle para um processo não linear com grau relativo unitário para estimar o erro de modelagem e assim mitigar seus efeitos na malha de controle EFL(Oliveira et al., 2017). Baseado nessa topologia de controle, (Oliveira et al., 2019) sugere uma nova estrutura de controle que faz uso do princípio da equivalência certa (certainty equivalente principle) para estimar um sinal de compensação e reduzir o efeito de pertubações originadas por erros de modelagem.

Esse trabalho analisa e avalia o controlador de linearização por realimentação granular robusto (robust granular feedback linearization - RGFL) apresentado em (Oliveira et al., 2019), mas utilizando o algoritmo de aprendizagem eTS ao invés do original ePL. Esse controlador é utilizado para controlar um pêndulo invertido e fazer que seu estado siga uma trajetória especificada. O pêndulo invertido tem sido adotado como benchmark por diversos trabalhos (Park et al., 2003), (Wang, 1994), (Slotine and Li, 1991), (Khalil, 2002). Os resultados são quantificados usando a integral do valor absoluto do erro - IAE, integral da variabilidade do erro - IVE e integral da variabilidade do sinal de controle - IVU como índices de desempenho. A análise dos resultados de simulação mostram que, similarmente ao que ocorre com o ePL, o algoritmo eTS torna a linearização por realimentação granular mais robusta a erros de modelagem do que o EFL.

Este trabalho está organizado da seguinte forma. A Seção 2 apresenta uma revisão dos métodos e técnicas utiliza- das neste artigo, o algoritmo evolutivo Takagi-Sugeno, a linearização por realimentação, e o controlador de linearização por realimentação granular robusto. A Seção 3 desenvolve o controlador granular robusto com o algoritmo eTS. Os resultados obtidos para o problema de seguimento de referência por meio de simulação do sistema do pêndulo invertido são apresentados na Seção 4. Por fim, a Seção 5 resume as conclusões e trabalhos futuros.

\section{PRELIMINARES}

\subsection{Algoritmo evolutivo Takagi-Sugeno}

O algoritmo evolutivo Takagi-Sugeno (eTS) (Angelov and Filev, 2004) baseia-se em um método de agrupamento recursivo adaptativo que permite atualizar e/ou criar grupos autonomamente em tempo real, diferentemente do método original de Takagi e Sugeno (Lughofer, 2011). No eTS cada grupo no espaço de dados corresponde a uma regra fuzzy funcional da forma:

$$
\begin{aligned}
\mathfrak{R}_{i}: \mathbf{S E ~} \mathbf{z}(k) \text { é } X_{i} \text { ENTÃ O } y_{i}(k) & =\gamma_{i}\left[\begin{array}{ll}
1 & \mathbf{z}(k)
\end{array}\right]^{T} \\
i & =1, \cdots, c(k),
\end{aligned}
$$

em que $\mathfrak{R}_{i}$ é a $i$-ésima regra, $\mathbf{z}(k) \in[0,1]^{p}$ é a entrada no $k$-ésimo passo, $X_{i}$ é um conjunto fuzzy, $\gamma_{i} \in \mathbb{R}^{p+1}$ é o vetor de parâmetros, $y_{i}$ é a saída da $i$-ésima regra, $c(k)$ o número de regras fuzzy no $k$-ésimo passo e $p$ a dimensão do espaço de entrada.

O processo de aprendizado do eTS baseia-se na avaliação do potencial do dado de entrada para atualizar e modificar a base de regras fuzzy (Angelov et al., 2004a). Esse parâmetro oferece uma medida da aproximação espacial entre o dado e o centro focal da regra fuzzy (Angelov, 2013). Nesse sentido o processo inicia-se com a aquisição do primeiro dado de entrada $\mathbf{z}(1)$, o qual é definido como o centro focal do primeiro cluster, $\mathbf{x}_{1}$. São também inicializados o potencial do cluster, $P_{1}=1$, e os parâmetros do modelo local de saída da regra fuzzy, sendo: a matriz de covariância, $Q_{1}=\Omega \mathbf{I}$, em que $\Omega$ é um número positivo de grande valor numérico, e o vetor de parâmetros $\gamma_{1}=\left[\begin{array}{llll}0_{1} & 0_{2} & \cdots & 0_{p+1}\end{array}\right]$. Após a criação do primeiro cluster, o potencial dos dados de entrada subsequentes são computados recursivamente (Angelov and Filev, 2004) usando:

em que

$$
P_{z}(k)=\frac{k-1}{(k-1)(\vartheta(k)+1)+\sigma(k)-2 v(k)}
$$

$$
\begin{gathered}
\vartheta(k)=\sum_{j=1}^{p+1}\left(z^{j}(k)\right)^{2} ; \quad \sigma(k)=\sum_{i=1}^{k-1} \sum_{j=1}^{p+1}\left(z^{j}(i)\right)^{2} ; \\
v(k)=\sum_{j=1}^{p+1} z^{j}(k) \beta^{j}(k) ; \quad \beta^{j}(k)=\sum_{i=1}^{k-1} z^{j}(i),
\end{gathered}
$$

e $j=1, \cdots, p$. Observe que o cálculo de $\vartheta(k)$ e $v(k)$ são realizados a partir do dado de entrada atual, enquanto $\beta^{j}(k)$ e $\sigma(k)$ são computados recursivamente (Lughofer, 2011), tal que:

$$
\begin{aligned}
& \sigma(k)=\sigma(k-1)+\sum_{j=1}^{p+1}\left(z^{j}(k-1)\right)^{2} \\
& \beta^{j}(k)=\beta^{j}(k-1)+z^{i}(k-1)
\end{aligned}
$$


A entrada de um novo dado $(\mathbf{z}(k))$ influencia diretamente o potencial de todos os grupos (Angelov et al., 2004a). O potencial de cada grupo é atualizado recursivamente usando:

$$
P_{x_{i}^{*}}(k)=\frac{(k-1) P_{x_{i}^{*}}(k-1)}{k-2+P_{x_{i}^{*}}(k-1)\left(1+\sum_{j=1}^{p+1}\left(d_{i(k-1)}^{j}\right)^{2}\right)}
$$

em que $P_{x_{i}^{*}}(k)$ é o potencial do centro do cluster no $k$ ésimo passo, e $d_{i(k-1)}^{j}=x_{i}^{j}-z_{k}^{j}$ é a projeção da distância entre o dado de entrada $\mathbf{z}(k)$ e os centros de grupo $\mathbf{x}_{i}$ sobre os eixos $\mathbf{x}$.

O potencial do dado de entrada, $P_{z}(k)$, é comparado com o potencial dos grupos, $P_{x_{i}^{*}}(k)$, para que seja avaliado a viabilidade de criar novas regras, ou atualizar uma regra existente, seguindo critérios que podem ser vistos com mais detalhes em (Angelov et al., 2004a), (Lughofer, 2011), (Angelov, 2013). Desse modo, se uma condição de atualização for satisfeita, então o dado de entrada ajusta o centro do cluster mais próximo, e o vetor de parâmetros dos consequentes é atualizado com o auxílio do algoritmo dos quadrados mínimos recursivo (Recursive Least Square - RLS) (Ljung, 1999). Por outro lado, se a condição de criação de regras for satisfeita, então o dado torna-se o centro de um novo grupo e a regra correspondente é inserida na base de regras. Se nenhuma das duas condições forem satisfeitas, então o dado de entrada é descartado e a base de regras permanece a mesma.

A saída do algoritmo eTS, $y(k)$, é determinada pela média ponderada das saídas de cada regra, $y_{i}(k)$, (Angelov et al., 2004b), isto é:

$$
y(k)=\frac{\sum_{i=1}^{c(k)} \mu_{i}(k) \gamma_{i}[1 \mathbf{z}(k)]^{T}}{\sum_{i=1}^{c(k)} \mu_{i}(k)}
$$

em que $\mu_{i}(k)$ é o grau de ativação da $i$-ésima regra fuzzy, computado com auxílio da função Gaussiana:

$$
\mu_{i}(k)=e^{-4 \frac{\left\|\mathbf{z}(k)-\mathbf{x}(k){ }_{i}^{*}\right\|^{2}}{r^{2}}}
$$

em que $r$ é uma constante positiva que regula a zona de influência da regra.

\subsection{Linearização por Realimentação Exata}

Considere a classe de sistemas não lineares com uma entrada e uma saída (Single-Input Single-Output - SISO), dada por:

$$
\begin{aligned}
x^{(n)} & =f(\mathbf{x})+g(\mathbf{x}) u \\
y & =x
\end{aligned}
$$

em que $\mathbf{x}=\left[\begin{array}{llll}x & \dot{x} & \cdots & x^{(n-1)}\end{array}\right]^{T} \in \mathrm{D} \subseteq \mathbb{R}^{n}$ é o vetor de estados, $u$ e $y$ são respectivamente a entrada e saída do sistema, $x^{(n)}$ é a $n$-ésima derivada do estado $x, f(\mathbf{x})$ e $g(\mathbf{x})$ são funções não lineares suaves ${ }^{1}$ pertencentes ao conjunto $\mathrm{D} \subseteq \mathbb{R}^{n}$. Por sua vez, o sistema (6) é controlável somente se $\bar{g}(\mathbf{x}) \neq 0 \quad \forall \mathbf{x} \in \mathrm{D} \subseteq \mathbb{R}^{n}$ (Khalil, 2002), o que resulta na lei de controle linearizante descrita por:

$$
u=\frac{1}{g(\mathbf{x})}[v-f(\mathbf{x})]
$$

em que $v$ é um sinal de controle obtido a partir de uma lei de controle linear, como por exemplo, a realimentação

1 Considera-se como funções suaves aquelas que são infinitamente diferenciáveis (Sastry, 1999). de estados, $v=K \mathbf{x}$, com $K \in \mathbb{R}^{(1 \times n)}$ (Chen, 1999). Desse modo, ao aplicar (7) em (6), as não linearidades do sistema são canceladas (Isidori, 1995), tal que:

$$
x^{(n)}=K \mathbf{x} .
$$

Portanto, se $K$ é Hurwitz (Ho et al., 1998), então todos os polos da malha de controle estão estritamente no semiplano complexo esquerdo, o que significa uma dinâmica exponencialmente estável para o sistema em malha fechada, isto é, $x(t) \rightarrow 0$ quando $t \rightarrow \infty$ (Slotine and Li, 1991).

\subsection{Controlador de Linearização por Realimentação Granular Robusto}

O controlador de linearização por realimentação granular robusto (robust granular feedback linearization - RGFL) foi originalmente proposto por Oliveira et al. em (Oliveira et al., 2019) onde sugere-se a inclusão de um sinal de compensação à malha de controle da linearização por realimentação para mitigar os efeitos causados pela diferença entre o modelo usado no projeto da lei de controle linearizante e o sistema real. Nessa abordagem, o sinal de compensação é estimado com o auxílio do algoritmo de aprendizagem participativa - ePL (Lima et al., 2010). Nesse sentido, assume-se que os erros de modelagem das funções $f(\mathbf{x})$ e $g(\mathbf{x})$ do sistema não linear (6) são aditivos, isto é:

$$
\begin{aligned}
& f(\mathbf{x})=f_{n}(\mathbf{x})+\Delta f(\mathbf{x}), \\
& g(\mathbf{x})=g_{n}(\mathbf{x})+\Delta g(\mathbf{x}),
\end{aligned}
$$

em que $f_{n}(\mathbf{x})$ e $g_{n}(\mathbf{x})$ são as funções nominais do sistema (conhecidas precisamente) e $\Delta f(\mathbf{x})$ e $\Delta g(\mathbf{x})$ são os termos que representam as partes desconhecidas (erros) de $f(\mathbf{x})$ e $g(\mathbf{x})$. Assim, ao observar o efeito promovido pela presença do erro de modelagem (9) na malha fechada de controle, Oliveira et al. propõem a alteração da lei de controle (7), o que resulta em:

$$
u_{s}=\underbrace{\frac{1}{g_{n}(\mathbf{x})}\left[v-f_{n}(\mathbf{x})-g_{n}(\mathbf{x}) u_{r}\right]}_{u_{e}}-\underbrace{\frac{1}{g_{n}(\mathbf{x})} \hat{\Delta}}_{u_{c}}+u_{r} .
$$

em que $u_{s}$ é o sinal de controle compensado, $u_{e}$ o sinal de controle para correção do erro de trajetória $\mathbf{e}=\mathbf{x}-\mathbf{r}$, $\operatorname{com} \mathbf{e}=\left[\begin{array}{lll}e \dot{e} \cdots e^{(n-1)}\end{array}\right]^{T}$ e $\mathbf{r}=\left[\begin{array}{llll}r & \dot{r} & \cdots & r^{(n-1)}\end{array}\right]^{T}, u_{c} \mathrm{o}$ sinal de controle para compensação do erro de modelagem e $\hat{\Delta}$ o valor estimado para as incertezas, computado com o uso do algoritmo ePL. Desse modo, ao aplicar (10) em (6), considerando a presença de incertezas (9), obtêm-se a dinâmica para a malha fechada, dada por:

$$
e^{(n)}=v+\Delta-\hat{\Delta}-r^{(n)} \text {. }
$$

Assume-se que a perturbação estimada com o algoritmo ePL, seja tal que: $\hat{\Delta}=\boldsymbol{\Delta}+w$ em $w(t) \rightarrow 0$ quando $t \rightarrow \infty$, descreve o erro de estimação do algoritmo ePL. Além disso, considerando o problema de seguimento de referência, defini-se a lei de controle linear, tal que: $v=K \mathbf{e}+r^{(n)}$, o que resulta na dinâmica do sistema em malha fechada, definida por:

$$
e^{(n)}=K \mathbf{e}-w
$$

a qual pode ser reescrita como:

$$
\dot{\mathbf{e}}=A_{c} \mathbf{e}+B_{c} w,
$$

em que 


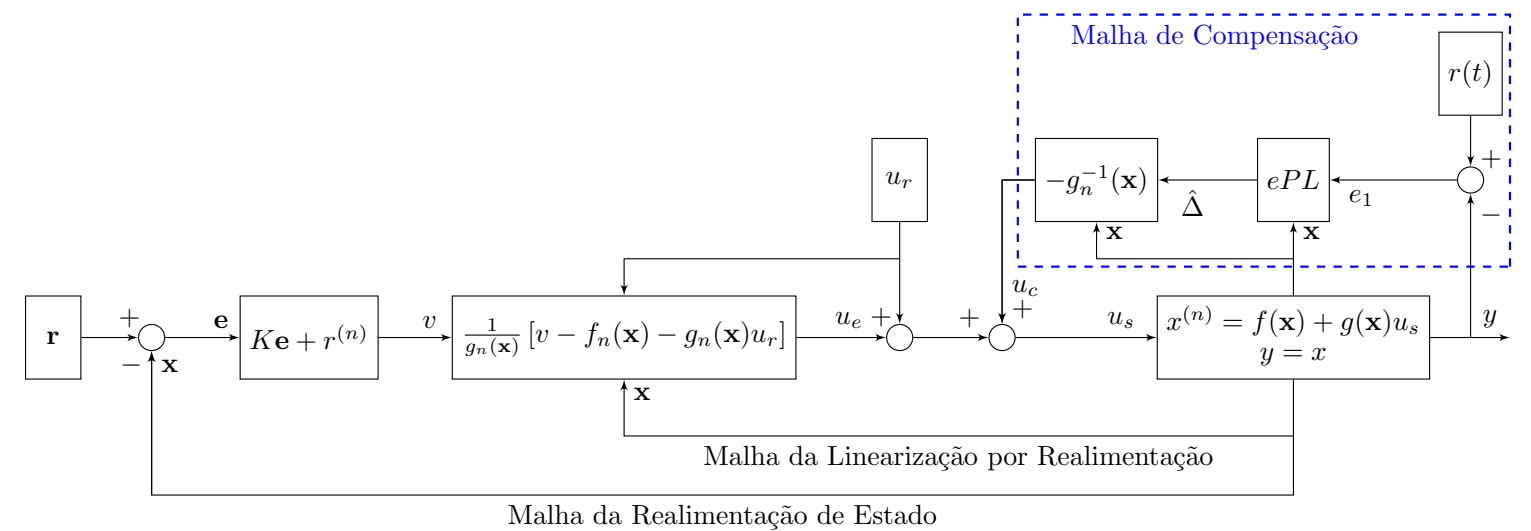

Figura 1. Linearização por realimentação granular robusta - RGFL.

$$
A_{c}=\left[\begin{array}{cccc}
0 & 1 & \cdots & 0 \\
0 & 0 & \cdots & 0 \\
\vdots & \vdots & \ddots & \vdots \\
0 & 0 & \cdots & 1 \\
k_{n} & k_{n-1} & \cdots & k_{1}
\end{array}\right] \text { e } B_{c}=\left[\begin{array}{c}
0 \\
0 \\
\vdots \\
0 \\
-1
\end{array}\right]
$$

A topologia do controlador RGFL resultante é mostrada na Figura 1.

\section{CONTROLADOR GRANULAR ROBUSTO ETS}

Em sua versão original, o controlador RGFL utiliza o mecanismo de aprendizagem partipativa ePL para construir e atualizar a base de regras fuzzy cujo propósito é estimar o valor do erro $\hat{\Delta}(k)$ e minimizar seus efeitos na malha fechada de controle. Contudo, neste trabalho avaliase o desempenho da topologia proposta para o controlador RGFL quando o mecanismo de apredizado, ou seja, a construção e atualização da base de regras, é implementado com auxílio do algoritmo eTS ao invés do ePL original.

Nessa perspectiva, os dados de entrada do algoritmo de aprendizagem são o estado $(\mathbf{x})$ e do erro de saída do sistema $\left(e_{1}\right)$. As regras fuzzy funcionais são da seguinte forma:

$$
\text { SE } \mathbf{z}(k) \text { é } X^{i} \text { ENTÃO } y_{i}(k)=\gamma^{i}(k) \xi(k)+K_{p} e_{1}
$$

em que $X^{i}$ é o vetor de funções de pertinência do antecedente da $i$-ésima regra fuzzy e $\gamma^{i}(k)$ o vetor de parâmetros do consequente. Os parâmetros do consequente são computados usando o algoritmo RLS, $K_{p}$ é um parâmetro de projeto, e $\xi(k)$ um vetor construído a partir dos estados do sistema e erros de modelagem estimadas em passos anteriores, isto é:

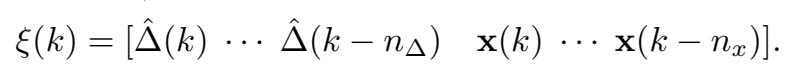

O Algoritmo 1 mostra o pseudocódigo correspondente ao controlador RGFL com aprendizado eTS.

A avaliação feita neste trabalho considera diferentes cenários (condições para atualização de regras existentes e condições para criação de novas regras), conforme resume a Tabela 1. Esses cenários são estabelecidos na literatura: o cenário $A$ proposto em (Angelov and Filev, 2004), os cenários $B$ e $C$ em (Ramos and Dourado, 2003), e os cenários $D, E, F, G$ em (Angelov et al., 2004a).

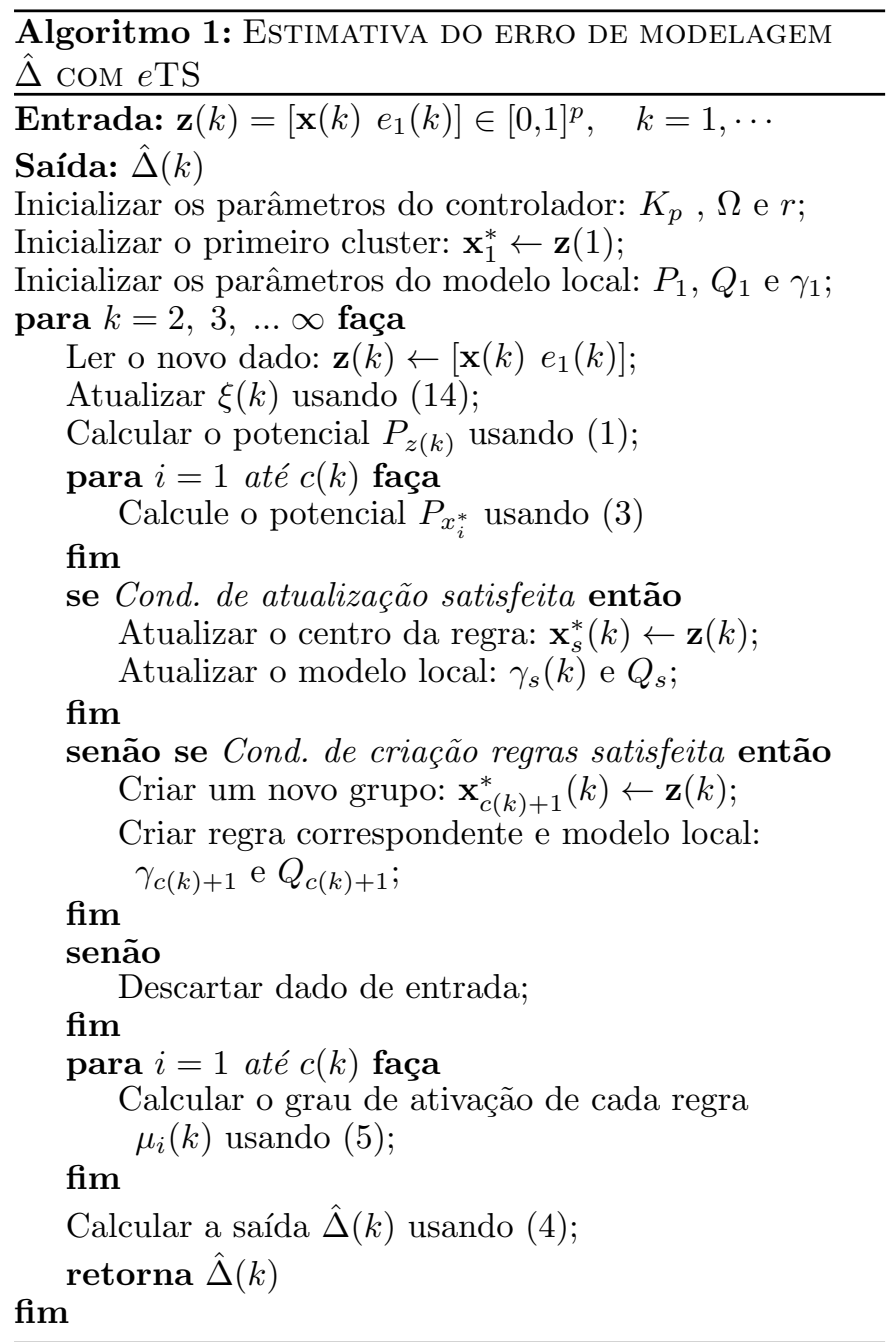

\section{RESULTADOS DE SIMULAÇÃO}

Esta seção apresenta os resultados de simulação para o sistema de controle de posição de um pêndulo invertido. O pêndulo invertido, mostrado na Figura 2, é utilizado por diversos autores (Slotine and Li, 1991), (Wang, 1994), (Park et al., 2003) para avaliar abordagens de controle que envolve a linearização por realimentação adaptativa. A dinâmica do pêndulo invertido é descrita pelas equações diferenciais: 
Tabela 1. Cenários de avaliação e condições para atualizar e criar novas regras pelo eTS.

\begin{tabular}{|c|c|c|}
\hline Cenário & Condição criação de novas regras & Condição atualização de regras existentes \\
\hline $\mathrm{A}$ & $P_{z}>0.5 P_{\max }$ & $P_{z}>0.15 P_{\max } e \frac{d_{\min }}{r}+\frac{P_{\max }}{P_{z}}<1$ \\
\hline $\mathrm{B}$ & $P_{z}>P_{\operatorname{mean}}$ & $P_{z}>P_{\operatorname{mean}} e \frac{d_{\min }}{r}+\frac{P_{\max }}{P_{z}}<1$ \\
$\mathrm{C}$ & $P_{z}>P_{\max }$ & $P_{z}>P_{\max } e \frac{d_{\min }}{r}+\frac{P_{\max }}{P_{z}}<1$ \\
\hline $\mathrm{D}$ & $P_{z}>P_{\max }$ & $P_{z}>P_{\max } e \frac{d_{\min }}{r}<0.5$ \\
$\mathrm{E}$ & $P_{z}>P_{\max }$ ou $0.5 P_{\max }<P_{z}<0.675 P_{\max }$ & $\left(P_{z}>P_{\max }\right.$ ou $\left.0.5 P_{\max }<P_{z}<0.675 P_{\max }\right) e \frac{d_{\min }}{r}<0.5$ \\
$\mathrm{~F}$ & $P_{z}>P_{\max }$ ou $P_{z}<P_{\min }$ & $\left(P_{z}>P_{\max }\right.$ ou $\left.P_{z}<P_{\min }\right) e \frac{d_{\min }}{r}<0.5$ \\
$\mathrm{G}$ & $P_{z}>P_{\max }$ ou $P_{z}<P_{\min }$ & $\left(P_{z}>P_{\max } e \frac{d_{\min }}{r}<0.5\right)$ ou $\left(P_{z}<P_{\min } e \frac{d_{\min }}{r}<0.85\right)$ \\
\hline
\end{tabular}

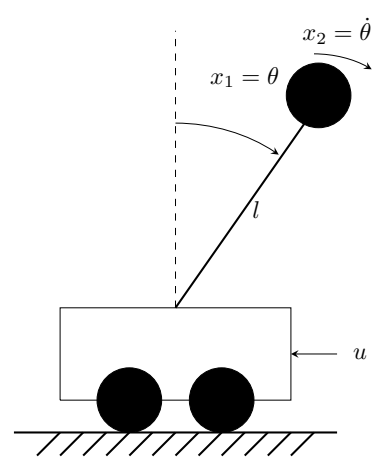

Figura 2. Pêndulo invertido.

$$
\begin{aligned}
& \dot{x}_{1}=x_{2} \\
& \dot{x}_{2}=\frac{g \sin x_{1}-\frac{m l x_{2}^{2} \cos x_{1} \sin x_{1}-\cos x_{1} u}{m_{c}+m}}{l\left(4 / 3-\frac{m \cos ^{2} x_{1}}{m_{c}+m}\right)}
\end{aligned}
$$

em que $x_{1}=\theta[\mathrm{rad}]$ é o ângulo do pêndulo com a normal, $x_{2}=\dot{\theta}[\mathrm{rad} / \mathrm{s}]$ é a velocidade ângular, $g=9,8 \mathrm{~m} / \mathrm{s}^{2}$ é a aceleração da gravidade, $m_{c}[k g]$ é a massa do carro, $m[k g]$ é a massa do pêndulo, $l[m]$ é o comprimento da haste do pêndulo, e $u[N]$ é a força necessária para manter o pêndulo na posição desejada, sendo este o sinal de controle para o sistema.

As simulações foram realizadas com o auxílio do modelo discretizado do pêndulo via método de Euler, tal que:

$$
\begin{aligned}
& x_{1}(k+1)=x_{1}(k)+T x_{2}(k) \\
& x_{2}(k+1)=x_{2}(k)+T[f(\mathbf{x}(k))+g(\mathbf{x}(k)) u(k)]
\end{aligned}
$$

em que $T$ é o período de amostragem, $f(\mathbf{x}(k))$ e $g(\mathbf{x}(k))$ são as funções com incertezas nos parâmetros. Os parâmetros de modelagem do sistema foram definidos conforme sugerido em (Park et al., 2003), em que: $m_{c}=1 \mathrm{~kg}, \mathrm{~m}=0.1 \mathrm{~kg}$ e $l=0.5 \mathrm{~m}$. Já a inclusão de incertezas nos parâmetros se da por: $m_{\text {inc }}=m\left(1+\varsigma_{0}\right)$ e $l_{\text {inc }}=l\left(1+\varsigma_{1}\right)$, em que $\varsigma_{i}$ para $i=0,1$ são as incertezas dos parâmetros. Além disso, adotou-se como referência para a saída do sistema a trajetória gerada pela função: $r(t)=(\pi / 30) \sin (t)$. Para tal, a lei de controle compensada (10) é reescrita em sua forma discreta, tal que:

$$
\begin{aligned}
u_{s}(k)=\frac{1}{g_{n}(\mathbf{x}(k))}[v(k)- & \left.f_{n}(\mathbf{x}(k))-g_{n}(\mathbf{x}(k)) u_{r}(k)\right] \\
& -\frac{1}{g_{n}(\mathbf{x}(k))} \hat{\Delta}(k)+u_{r}(k)
\end{aligned}
$$

em que $v(k)$ é o sinal de controle linear, produzido pela malha de realimentação de estados, com ganho $K=$ [-2 -1] (Park et al., 2003), e $u_{r}(k)$ dado por:

$$
u_{r}(k)=\frac{r^{(n)}-f_{n}(\mathbf{r}(k))}{g_{n}(\mathbf{r}(k))} .
$$

Para avaliar o comportamento do sistema (15) em malha fechada com o controlador RGFL usando o algoritmo eTS como mecanismo de aprendizado, foram considerados dois cenários distintos durante a realização das simulações. Inicialmente, considerou-se o sistema perfeitamente conhecido, ou seja, sem a presença de incertezas $\left(\varsigma_{0}=\right.$ $\left.0, \varsigma_{1}=0\right)$. Contudo, em um segundo cenário assumiu-se a presença de incertezas contantes nos parâmetros $m$ e $l$, sendo, $\varsigma_{0}=-0.2$ e $\varsigma_{1}=-0.15$, respectivamente. Os resultados obtidos para o sistema incerto, são apresentados no gráfico da Figura 3, seguindo o seguinte padrão de identificação: controlador EFL com linha tracejada azul, controlador RGFL com as condições (ver Tabela 1) A e C linha contínua azul, condições D e E linha traço-ponto azul, condição B linha pontilhada azul e condições F e G linha contínua preta. A trajetória usada como referência é representa pela linha tracejada preta.

Os resultados apresentados pela gráfico da Figura 3, foram obtidos configurando o eTS com seguintes valores para as constantes: $K_{p}=5$ e $\Omega=10000$. Ademais, adotouse o método de mínimo quadrados recursivo com fator de esquecimento $\lambda=0.98$, como ferramenta para atualização dos parâmetros do modelo local das regras. Por fim, conforme sugerido por (Ramos and Dourado, 2003) a região de influência da gaussiana, definida pelo parâmetro $r$, deve ser escolhida de modo a atenter ao intervalo $0,3 \leq$ $r \leq 0,5$. Portanto, avaliou-se o controlador sob as mesmas condições, para os seguintes valores de $r=\left[\begin{array}{lll}0,3 & 0,4 & 0,5\end{array}\right]$.

Ao analisar o gráfico da Figura 3, pode-se verificar que o controlador RGFL quando submetido as condições F e G não conseguiu atuar de modo a manter a estabilidade do sistema, fazendo com que o mesmo atingisse seu alcance limite $\theta=\frac{\pi}{2}\left[\begin{array}{ll}-1 & 1\end{array}\right]$. Já quando o controlador é submetido a condição $\mathrm{B}$, esse atua de modo a manter o pêndulo na posição desejada, porém observa-se que ao longo do tempo o erro $e_{1}$ está em constante crescente, o que sugere que o sistema pode alcançar em uma janela maior de tempo a instabilidade. Por outro lado, quando usa-se as condições D e E o controlador mantém a estabilidade do sistema, porém a magnitude do erro mantém-se elevada. Por fim, observa-se que as condições A e C consegue além de manter a estabilidade do sistema, reduzir ao longo tempo o erro de seguimento da referência. É importante observar, que o controlador EFL em sua forma original não percebe o erro de modelagem, portanto não atua a fim de reduzir seus efeitos, o que resulta em um erro constante ao longo da simulação. 


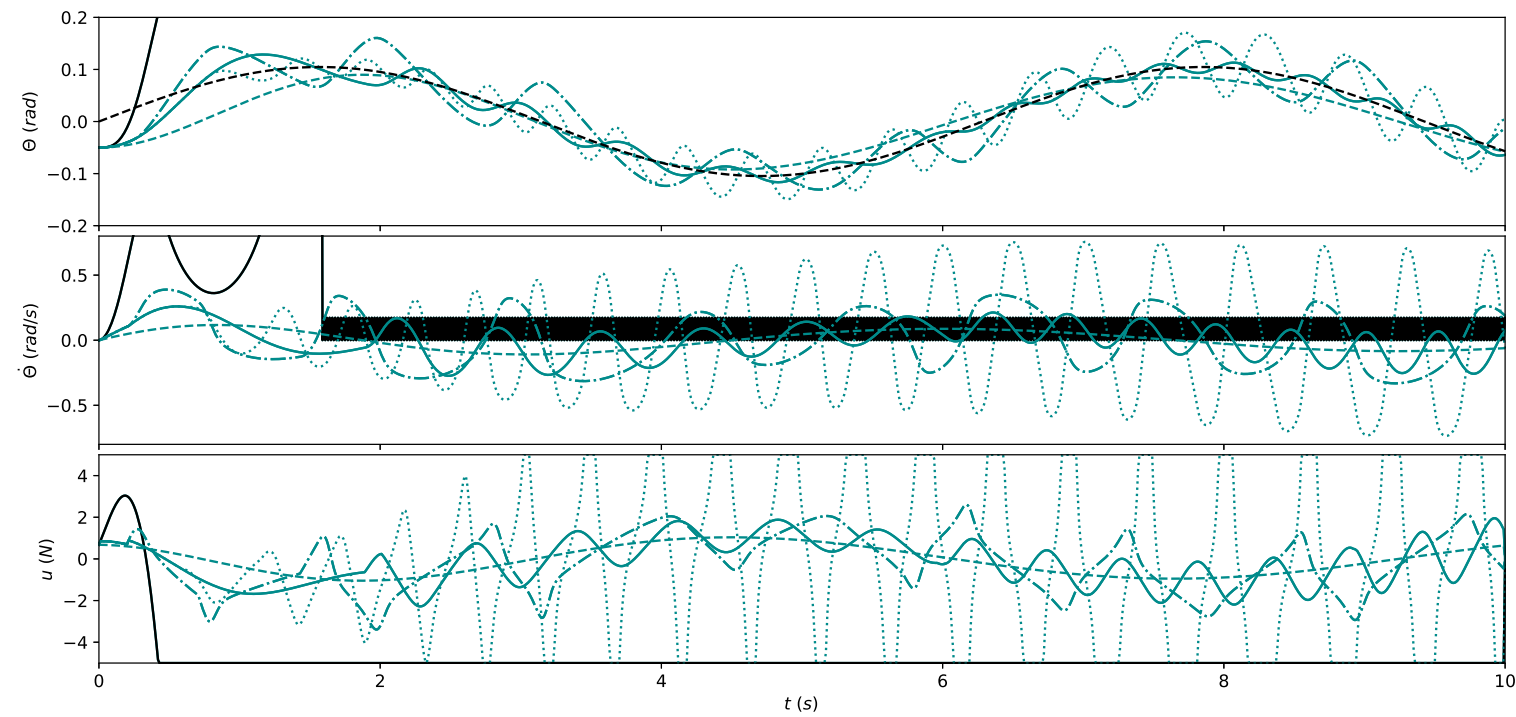

Figura 3. Comportamento do pêndulo com o controlador RGFL eTS e $r=0,3$.

A performance do controlador é quantificada com o auxílio dos indíces: integral do valor absoluto do erro $-I A E=$ $\int_{t_{0}}^{t_{f}}|e(t)| d t$, integral da variabilidade do erro $-I V E=$ $\sqrt{\frac{1}{t_{f}-t_{0}} \int_{t_{0}}^{t_{f}}(e(t)-\bar{e})^{2} d t}$ e integral da variabilidade do sinal de controle $-I V U=\sqrt{\frac{1}{t_{f}-t_{0}} \int_{t_{0}}^{t_{f}}(u(t)-\bar{u})^{2} d t}$. Esses indíces foram normalizados em relação ao desempenho obtido com o controlador EFL, $I_{x_{\text {norm }}}=\frac{I_{x}}{I_{E F L}}$. Em que $I_{x_{n \text { orm }}}$ é o indíce normalizado, $I_{x}$ é o índice a ser normalizado e $I_{E F L}$ é o indíce obtido com o controlador EFL. Desse modo, o desempenho do controlador RGFL será melhor que aquele alcançado com o controlador EFL se $I_{x_{\text {norm }}}<1$. Caso contrário o desempenho do controlador RGFL será pior. Os resultados são apresentados na Tabela 2 e Figura 4. Note que na Figura 4, quanto mais próximo do centro, melhor o desempenho.

Tabela 2. Desempenho do controlador RGFL eTS.

\begin{tabular}{|c|c|c|c|c|c|}
\hline \multicolumn{6}{|c|}{ Sistema Nominal } \\
\hline Cenário & $r$ & regras & $\overline{I I A E}$ & $I V E$ & $I V U$ \\
\hline \multirow{3}{*}{$\mathrm{A} / \mathrm{C}$} & 0.03 & 24 & 0.948 & 0.761 & 1.407 \\
\hline & 0.04 & 23 & 0.973 & 0.768 & 1.435 \\
\hline & 0.05 & 23 & 0.998 & 0.78 & 1.484 \\
\hline \multirow{3}{*}{ B } & 0.03 & 122 & 2.077 & 1.498 & 4.243 \\
\hline & 0.04 & 102 & 2.612 & 1.903 & 4.581 \\
\hline & 0.05 & 92 & 3.158 & 2.34 & 4.805 \\
\hline \multirow{3}{*}{$\mathrm{D} / \mathrm{E}$} & 0.03 & 1 & 2.58 & 1.768 & 1.87 \\
\hline & 0.04 & 1 & 3.348 & 2.278 & 2.342 \\
\hline & 0.05 & 1 & 3.957 & 2.703 & 2.803 \\
\hline \multicolumn{6}{|c|}{ Sistema com Erro de Modelagem } \\
\hline \multirow{3}{*}{$\mathrm{A} / \mathrm{C}$} & 0.03 & 28 & 0.651 & 0.697 & 1.583 \\
\hline & 0.04 & 27 & 0.655 & 0.701 & 1.585 \\
\hline & 0.05 & 26 & 0.665 & 0.705 & 1.586 \\
\hline \multirow{3}{*}{ B } & 0.03 & 119 & 1.526 & 1.422 & 4.975 \\
\hline & 0.04 & 111 & 2.048 & 1.928 & 5.550 \\
\hline & 0.05 & 95 & 2.451 & 2.347 & 5.798 \\
\hline \multirow{3}{*}{$\mathrm{D} / \mathrm{E}$} & 0.03 & 1 & 1.681 & 1.514 & 2.047 \\
\hline & 0.04 & 1 & 2.330 & 2.076 & 2.607 \\
\hline & 0.05 & 1 & 2.808 & 2.494 & 3.179 \\
\hline
\end{tabular}

Conforme mostra a Tabela 2, o controlador RGFL obteve desempenho superior quando submetido as condições A e C. Além disso, é importante observar que esse resultado foi obtido com o auxílio de um sistema fuzzy composto por 24 regras para o sistema nominal e 28 regras quando aplicado ao sistema incerto. Já na Figura 4 é possível verificar que o controlador RGFL sempre atua a fim de minimizar os efeitos da pertubação na malha fechada, com isso os valores obtidos para o índice IVU são superiores a um.

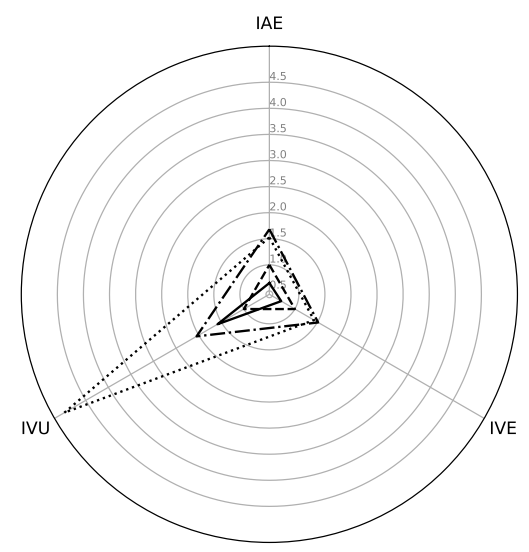

Figura 4. Desempenho do controlador RGFL eTS com $r=0,3$.

\section{CONCLUSÃO}

Este trabalho analisou e avaliou o controlador de linearização por realimentação granular robusto - RGFL com aprendizagem eTS. Esse controlador foi utilizado para verificar o desempenho quando no seguimento de referência em um pêndulo invertido. Os resultados foram quantificados usando os índices $I A E, I V E$ e $I V U$. A análise dos resultados mostra que o controlador RGFL com o algoritmo eTS aplicado ao sistema em estudo tem desempenho superior à linearização por realimentação exata. Contudo, torna-se necessário estudar e avaliar alterações na zona de influência induzida por $r$, assim como desenvolver condi- 
ções para permitir a análise e assegurar a estabilidade do sistema.

\section{AGRADECIMENTOS}

Os primeiros autores agradecem ao CEFET-MG e à FAPEMIG pelo projeto de iniciação científica 10546/2017, o apoio financeiro e o suporte técnico. O último autor é grato ao CNPq, Conselho Nacional de Desenvolvimento Científico e Tecnológico, processo 305906/2014-3 pelo apoio.

\section{REFERÊNCIAS}

Angelov, P. (2013). Autonomous Learning Systems: From Data Streams to Knowledge in Real-time. John Wiley \& Sons Ltd, 1 edition.

Angelov, P., Victor, J., Dourado, A., and Filev, D. (2004a). On-line evolution of Takagi-Sugeno fuzzy models. IFAC Proceedings Volumes, 37(16), 67 - 72. doi:https://doi. org/10.1016/S1474-6670(17)30852-2.

Angelov, P., Xydeas, C., and Filev, D. (2004b). On-line identification of mimo evolving Takagi- Sugeno fuzzy models. In 2004 IEEE International Conference on Fuzzy Systems (IEEE Cat. No.04CH37542), volume 1, 55-60 vol.1. doi:10.1109/FUZZY.2004.1375687.

Angelov, P.P. and Filev, D.P. (2004). An approach to online identification of Takagi-Sugeno fuzzy models. IEEE Transactions on Systems, Man, and Cybernetics, Part B (Cybernetics), 34(1), 484-498. doi:10.1109/ TSMCB.2003.817053.

Ataei, M., Esmaeilzadeh, R., and Alizadeh, G. (2005). Robust feedback linearization. In International Conference on Non-Linear Analysis, Non-Linear Systems an Chaos, 114 - 119. Sofia, Bulgaria.

Banerjee, S., Chakrabarty, A., Maity, S., and Chatterjee, A. (2011). Feedback linearizing indirect adaptive fuzzy control with foraging based online plant model estimation. Applied Soft Computing, 11(4), 3441 - 3450.

Chen, C. (1999). Linear System Theory and Design. Oxford University Press, New York, 3 edition.

Guillard, H. and Boulès, H. (2000). Robust feedback linearization. In Proceedings of the 14th International Symposium on Mathematical Theory of Networks and Systems. Paris.

Ho, M.T., Datta, A., and Bhattacharyya, S.P. (1998). An elementary derivation of the Routh-Hurwitz criterion. IEEE Transactions on Automatic Control, 43(3), 405409. doi:10.1109/9.661607.

Isidori, A. (1995). Nonlinear Control Systems. Springer, 3 edition.

Khalil, H. (2002). Nonlinear Systems. Prentice Hall, 3 edition.

Leite, D., Palhares, R., Campos, V., and Gomide, F. (2015). Evolving granular fuzzy model-based control of nonlinear dynamic systems. IEEE Transactions on Fuzzy Systems, 23(4), 923-938.

Lima, E., Hell, M., Ballini, R., and Gomide, F. (2010). Evolving Fuzzy Modeling Using Participatory Learning, 67-86. John Wiley \& Sons, Inc.

Ljung, L. (1999). System Identification: Theory for the User. Prentice-Hall, Inc., Upper Saddle River, NJ, USA, 2 edition.

Lughofer, E. (2011). Evolving Fuzzy Systems. Springer, 1 edition.
Oliveira, L., Leite, V., Silva, J., and Gomide, F. (2017). Granular evolving fuzzy robust feedback linearization. In Evolving and Adaptive Inteligent Systems. Ljubljana.

Oliveira, L., Leite, V., Bento, A., and Gomide, F. (2019). Robust granular feedback linearization. Paper aceppt to publish in the Fuzzy IEEE Conference.

Park, J. and Park, G. (2003). Robust adaptive fuzzy controller for non-affine nonlinear systems with dynamic rule activation. International Journal of Robust and Nonlinear Control, 13(2), 117-139. doi:10.1002/rnc.717.

Park, J.H., Seo, S.J., and Park, G.T. (2003). Robust adaptive fuzzy controller for nonlinear system using estimation of bounds for approximation errors. Fuzzy Sets and Systems, 133(1), $19-36$.

Precup, R., Bojan-Dragos, C., Hedrea, E., Rarinca, M., and Petriu, E.M. (2017). Evolving fuzzy models for the position control of magnetic levitation systems. In 2017 Evolving and Adaptive Intelligent Systems (EAIS), 1-6. doi:10.1109/EAIS.2017.7954839.

Ramos, J.V. and Dourado, A. (2003). Evolving TakagiSugeno fuzzy models. Technical report, Centre Inf. Syst. Adaptive Comput. Group, Univ. of Coimbra, Coimbra, Portugal, Tech. Rep.

Sastry, S. (1999). Nonlinear Systems - Analysis, Stability and Control. Springer, 1 edition.

Sastry, S.S. and Isidori, A. (1989). Adaptive control of linearizable systems. IEEE Transactions on Automatic Control, 34(11), 1123-1131. doi:10.1109/9.40741.

Silva, A.M., Caminhas, W.M., Lemos, A.P., and Gomide, F. (2013). Evolving neo-fuzzy neural network with adaptive feature selection. In 2013 BRICS Congress on Computational Intelligence and 11th Brazilian Congress on Computational Intelligence, 341-349. doi:10.1109/ BRICS-CCI-CBIC.2013.64.

Slotine, J. and Li, W. (1991). Applied Nonlinear Control. Prentice Hall, 1 edition.

Souza, L., Lemos, A., Caminhas, W., and Boaventura, W. (2012). Thermal modeling of power transformers using evolving fuzzy systems. Engineering Applications of Artificial Intelligence, 25(5), 980 - 988. doi:https:// doi.org/10.1016/j.engappai.2011.12.007.

Sugeno, M. and Takagi, T. (1983). Multi-dimensional fuzzy reasoning. Fuzzy Sets and Systems, 9(1-3), 313325.

Wang, L.. and Mendel, J.M. (1992). Fuzzy basis functions, universal approximation, and orthogonal least-squares learning. IEEE Transactions on Neural Networks, 3(5), 807-814. doi:10.1109/72.159070.

Wang, L.X. (1994). Adaptive Fuzzy Systems and Control: Design and Stability Analysis. Prentice Hall, 1 edition.

Wang, L. (1996). Stable adaptive fuzzy controllers with application to inverted pendulum tracking. IEEE Trans. Systems, Man, and Cybernetics, Part B, 26(5), 677-691.

Yang, Y., Feng, G., and Ren, J. (2004). A combined backstepping and small-gain approach to robust adaptive fuzzy control for strict-feedback nonlinear systems. IEEE Transactions on Systems, Man, and Cybernetics - Part A: Systems and Humans, 34(3), 406-420. doi: 10.1109/TSMCA.2004.824870. 